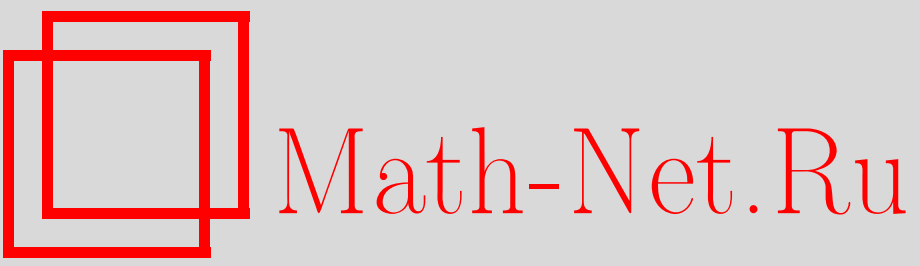

О. Е. Арсеньева, О геометрии конформно-полуплоских обобщенных эрмитовых поверхностей, УМH, 1997, том 52, выпуск 6, 149-150

DOI: https://doi.org/10.4213/rm896

Использование Общероссийского математического портала Math-Net.Ru подразумевает, что вы прочитали и согласны с пользовательским соглашением

http://www.mathnet.ru/rus/agreement

Параметры загрузки:

IP : 35.173 .137 .237

26 апреля 2023 г., 14:02:10 


\title{
О ГЕОМЕТРИИ КОНФОРМНО-ПОЛУПЛОСКИХ ОБОБЩЕННЫХ ЭРМИТОВЫХ ПОВЕРХНОСТЕЙ
}

\author{
О. Е. АРсЕНЬЕВА
}

Эрмитовой поверхностью назьвается 4-мерное почти эрмитово многообразие со знакоопределенной метрикой и интегрируемой почти комплексной структурой. Если $\{M, J, g=\langle\cdot, \cdot\rangle\}$ - эрмитова поверхность, то многообразие $M$ можно рассматривать как двумерное комплексное многообразие, снабженное эрмитовой метрикой $\ll X, Y \gg=\langle X, Y\rangle+\sqrt{-1}\langle X, J Y\rangle ; X, Y \in$ $\mathfrak{X}(M)$ (где $\mathfrak{X}(M)$ - модуль гладких векторных полей на $M$ ), т.е. как комплексный аналог римановых поверхностей, чем и объясняется название. Эрмитовы поверхности изучались с различных точек зрения многими авторами (см., например, [1]-[3]). Одна из важнейших особенностей геометрии эрмитовых поверхностей, как и всяких четырехмерных псевдоримановых многообразий, определяется тем, что группа Ли $S O(4, \mathbb{R})$ не является простой. Геометрическим следствием этого факта является сушествование канонического разложения модуля $\Lambda_{2}(M)$ 2-форм такого многообразия $M$ в прямую сумму подмодулей $\Lambda_{+}(M)$ автодуальных и $\Lambda_{-}(M)$ антиавтодуальных форм. Многообразие $M$ назьвается автодуальныцм (соответственно, антиавтодуальныцм), если ограничение эндоморфизма Вейля конформной кривизны на подмодуль $\Lambda_{-}(M)$ (соответственно, $\Lambda_{+}(M)$ ) равно нулю. Значение автодуальных и антиавтодуальных (иначе говоря, конформно полуплоских) многообразий определяется связью их геометрии с геометрией твисторного расслоения над такими многообразиями. Например, известная теорема Пенроуза-Атьи-Хитчина-Сингера [4] утверждает, что каноническая почти комплексная структура пространства твисторов 4-мерного ориентированного риманова многообразия интегрируема тогда и только тогда, когда это многообразие конформно полуплоско. В работе [5] Чен поставил проблему классификации конформно-полуплоских эрмитовых поверхностей. В направлении решения этой проблемы был получен ряд резултатов [6]. Однако существенные продвижения были получены лишь в случае келеровых поверхностей (т.е. 4-мерных келеровых многообразий) ([7]-[10]).

В настоящей работе получен ряд резултатов, касающихся геометрии конформно-полуплоских эрмитовых поверхностей как классического, так и гиперболического типа (обобщенных эрмитовых поверхностей), обобщающих известные в этом направлении резултаты Коды, Ито и других авторов.

ОПРЕДЕЛЕнИЕ. Обобщенной эрмитовой пов ерхностью называется тройка $\{M, J, g\}$, где $M-4$-мерное гладкое многообразие, $g=\langle\cdot, \cdot\rangle$ - псевдориманова метрика на $M, J$ - эндоморфизм касательного пучка многообразия $M$, назьваемый структурным әндоморфизмом, причем

$$
\text { 1) } \left.J^{2}=\alpha \text { id, } \quad \alpha= \pm 1 ; \quad 2\right)\langle J X, Y\rangle+\langle X, J Y\rangle=0 ; \quad X, Y \in \mathfrak{X}(M) .
$$

На всяком таком многообразии внутренним образом определены 2-форма $\Omega(X, Y)=\left\langle J^{3} X, Y\right\rangle$, называемая фундаментальной формой, а также 1-форма $\omega=\delta \Omega \circ J$, называемая формой Ли (здесь $\delta$ - оператор кодифференцирования). Заметим, что в случае $\alpha=-1$ и положительно определенной метрики мы приходим к понятию классической эрмитовой поверхности.

Теорема 1. Тензор Ричи $r$ автодуальной обобщенной әрмитовой поверхности $M$ комплексно линеен, и следовательно, определена форма Ричи $\rho(X, Y)=r\left(J^{3} X, Y\right)$. Эта форма автодуальна тогда и только тогда, когда $M$ - многообразие Эйнштейна, и антиавтодуальна тогда и только тогда, когда $M$ - многообразие нулевой скалярной кривизны.

Теорема 2. Автодуальная обобщенная эрмитова поверхность $М$ конформно плоска тогда и только тогда, когда

(1) $M-R K$-многообразие, т.е. его тензор $R$ Римана-Кристоффеля инвариантен относительно структурного әндоморфизма;

(2) $\delta \omega=\frac{1}{2}\|\omega\|^{2}-\frac{\varkappa}{3}$, где - скалярная кривизна многообразия. 
Теорема 3. Компактная автодуальная эрмитова поверхность $M$ неположительной скалярной кривизны конформно плоска тогда и только тогда, когда $M$ - келерово многообразие нулевой скалярной кривизны.

Теорема 4. Автодуальная обобщенная эрмитова поверхность является многообразием Эйнштейна тогда и только тогда, когда она является многообразием точечно постоянной голоморфной секционной кривизнь с. При этом $c=\frac{\varkappa}{6}-\frac{1}{8}\|\omega\|^{2}+\frac{1}{4} \delta \omega$.

Этот резултат существенно обобщает и усиливает известный резултат Коды [6], утверждающий, что автодуальная эрмитова поверхность Эйнштейна является многообразием точечно постоянной голоморффной секционной кривизны.

ТеОРема 5. Компактная автодуальная әрмитова поверхность постоянной неотричательной голоморфной секционной кривизны и неположительной скалярной кривизны локально голоморфно изометрична пространству $\mathbb{C}^{2}$, снабженному канонической келеровой структурой.

Теорема 6. Обобщенная әрмитова поверхность М антиавтодуальна тогда и только тогда, когда

(1) Внешний дифференциал ее формы Ли $\omega$ является антиавтодуальной 2-формой;

(2) $\delta \omega=\frac{1}{2}\|\omega\|^{2}-\frac{\varkappa}{3}$.

Этот результат существенно обобщает известный критерий Ито [9] антиавтодуальности келеровых поверхностей, а также соответствующий резултат автора для обобщенных келеровых поверхностей [10].

ТЕорема 7. Компактная обобщенная әрмитова поверхность $M$ с неизотропной 2-формой $d \omega$ (в частности, компактная әрмитова поверхность) антиавтодуальна тогда и только тогда, когда

(1) $M$ - локально конформно-келерово многообразие;

(2) $\delta \omega=\frac{1}{2}\|\omega\|^{2}-\frac{\varkappa}{3}$.

Этот резултат существенно обобщает и поясняет известный критерий Коды антиавтодуальности компактных эрмитовых поверхностей [6].

ТеОРема 8. Компактная антиавтодуальная эрмитова поверхность неположительной скалярной кривизны является келеровым многообразием нулевой скалярной кривизньр.

Теорема 9. Метрика компактной антиавтодуальной әрмитовой поверхности локально конформна метрике нулевой скалярной кривизны.

\section{СПИСОК ЛИТЕРАТУРЫ}

[1] Tricerri F., Vaisman I. // Math. Z. 1986. V. 192. P. 205-216. [2] Vaisman I. // Ann. Math. Pura ed Appl. 1982. V. 32. P. 1-18. [3] De Andres L. C., Cordero L. A., Fernandes M., Mencia J. J. // Geom. Dedicata. 1989. V. 29. P. 227-232. [4] Atiyah M.F., Hitchin N. J., Singer I. M.// Proc. Roy. London. 1978. V. A362. P. 425-461. [5] Chen B. Y. // Journ. Different. Geom. 1978. V. 13. P. 547-558. [6] Koda T. // Kodai Math. 1987. V. 10. P. 335-342. [7] Bourguignon J.-P. // Invent. Math. 1981. V. 63. P. 263-286. [8] Derdzinski A. // Comp. Math. 1983. V. 49. P. 405-433. [9] Itoh M. // Comp. Math. 1984. V. 51. P. 265-273. [10] Арсеньева О. Е. // Матем. сб. 1993. Т. 184. № 8. С. 137-148.

Московский педагогический 